\title{
Spontaneous Remission of Solitary-Type Infantile Myofibromatosis
}

\author{
Kazuhiro Kikuchia Riichiro Abe ${ }^{a}$ Satoru Shinkuma ${ }^{a}$ \\ Erika Hamasaka ${ }^{a}$ Ken Natsuga ${ }^{a}$ Hiroo Hata ${ }^{a}$ \\ Yasuki Tateishi $^{\mathrm{a}}$ Masahiko Shibata $^{\mathrm{a}}$ Yuki Tomita $^{\mathrm{a}}$ \\ Yukiko Abe $^{\text {a }}$ Satoru Aoyagi ${ }^{\text {a }}$ Makio Mukai \\ Hiroshi Shimizu ${ }^{a}$
}

aDepartment of Dermatology, Hokkaido University Graduate School of Medicine, Sapporo, and bDepartment of Pathology, Keio University School of Medicine, Tokyo, Japan

\section{Key Words}

Infantile myofibromatosis · Leiomyosarcoma · Solitary type

\begin{abstract}
Infantile myofibromatosis is a rare fibrous tumor of infancy. The cutaneous solitary type has typically an excellent prognosis. However, histologically, it is important to rule out leiomyosarcoma, which has a poor prognosis. The low frequency of mitosis was definitive for a diagnosis of infantile myofibromatosis. We present a cutaneous solitary-type case of infantile myofibromatosis. Following incisional biopsy, the tumor remitted spontaneously.
\end{abstract}

\section{Introduction}

Infantile myofibromatosis is a benign fibrous tumor of infancy and was first described by Stout in 1954 [1]. In most cases, it is present at birth, and in $90 \%$ of cases, the tumor appears within the first 2 years of life $[2,3]$. The prognosis is excellent in the solitary type, which is limited in the skin, muscle, and subcutaneous lesions [2-4]. In contrast, the multicentric form of infantile myofibromatosis, which has visceral involvement, can be life-threatening $[4,5]$. The solitary type is usually benign and the recurrence rate is low at $10 \%$. Therefore, surgical excision is recommended $[2,6]$. 
We present a case of a 3-week-old girl showing features of infantile myofibromatosis (solitary type). Excision was performed and no recurrence was detected in 24 months' follow-up.

\section{Case Report}

A 3-week-old, otherwise healthy Japanese girl had a solid, red-colored, cutaneous nodule on left shoulder. The nodule had a central concavity with a crust on the surface and measured $20 \times 21 \mathrm{~mm}$ in diameter (fig. 1).

Physical examination and CT imaging of the head, chest, abdomen and pelvis revealed no additional lesions. No infiltration of the tumor into the muscle was identified by MRI imaging (fig. 2). Incisional biopsy was performed when the patient was 4 months old. The specimen showed multifocal sclerotic dermal nodules. The nodules were composed of spindle cells with round or oval nuclei and eosinophilic cytoplasm. Delicate bundles of eosinophilic fibers separated the cellular aggregates (fig. $3 \mathrm{a}, \mathrm{b}$ ). A diagnosis of infantile myofibromatosis, leiomyoma, leiomyosarcoma, histiocytoma, or other sarcoma was suggested. Spindle cells expressed smooth muscle actin (fig. 3c), but not caldesmon, desmin or S100 protein (not shown). The mitotic figures were very infrequent [ 6 mitoses per 10 low-power images $(40 \times)]$. These results were confirmed to be consistent with infantile myofibromatosis. The tumor gradually regressed until it completely disappeared 24 months after biopsy.

\section{Discussion}

Infantile myofibromatosis usually develops at birth or during the first years of life. Chung and Enzinger found the median age at presentation to be 3 months [2]. A slight male predominance among patients with both the solitary and multicentric variants was noted by Wiswell et al. [7]. Most cases of infantile myofibromatosis are solitary nodules, accounting for up to $70 \%$ of cases in one study [2], and up to $80 \%$ in another series [4]. The prognosis is excellent in the solitary type [2-4]. In the case of solitary-type infantile myofibromatosis, spontaneous regression can be expected [3,4]. In contrast, a quarter of the cases with the multicentric form may have visceral involvement and can be lifethreatening $[2,4,5]$. The solitary type of infantile myofibromatosis is usually benign and is typically found in the dermis, subcutis, or deep soft tissues. The distribution is predominantly on the head, neck, and trunk like our case. Involvement of the extremities is reported to be rare [2]. Solitary infantile myofibromatosis on an upper extremity accounted for only $13.3 \%$ in one study of 45 cases [8].

The histologic hallmark of infantile myofibromatosis is an un-encapsulated, wellcircumscribed lobule of peripheral spindle cells, which bear a close resemblance to smooth muscle $[9,10]$. Often there is a central area of hemangiopericytoma-like small rounded cells surrounding blood vessels $[11,12]$. This combination of features gives infantile myofibromatosis its recognizable biphasic appearance, though the hemangiopericytoma-like appearance was not detected in this case. The presence of smooth muscle actin in the spindle cells indicates the diagnosis of infantile myofibromatosis or leiomyosarcoma. Considering the difference in prognosis, it was necessary to rule out leiomyosarcoma [13] in this case. While at least 1 mitotic cell per field in high-power $(\times 200$ or $\times 400)$ fields is detected in leiomyosarcoma [14], very infrequent mitotic figures [6 mitotic cells per 10 low-power $(\times 40)$ fields] were observed, which definitively indicated infantile myofibromatosis in this case. 
Previously, radical excision had been advocated as the treatment of choice, because it had been believed that the solitary form gave rise to multiple nodules with potential visceral involvement by metastases [15]. However, it is now more probable that the solitary and multicentric forms are distinct entities and that the solitary form remains localized and can regress [15]. Therefore, a wait-and-see approach has been suggested more recently as a treatment option [15]. However, in our patient, the decision was ultimately made to treat with surgical removal to exclude a diagnosis of leiomyosarcoma, which would have had a poor prognosis. The nodule disappeared completely after excision. The course was consistent with previous reports of solitary-type infantile myofibromatosis $[2,4,15]$, and supports our histological diagnosis.

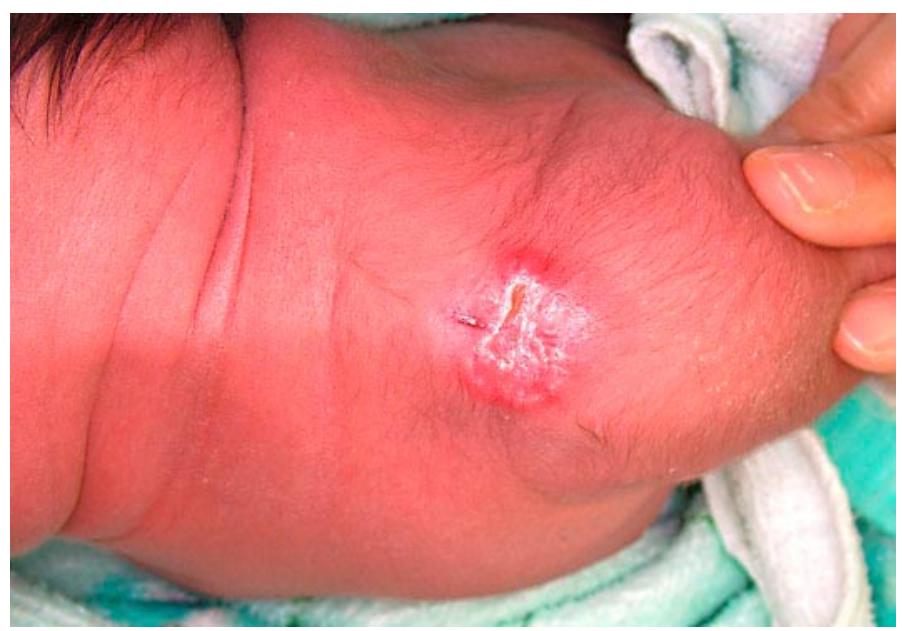

Fig. 1. Solid, red-colored subcutaneous nodule with a central concavity on the left shoulder. 


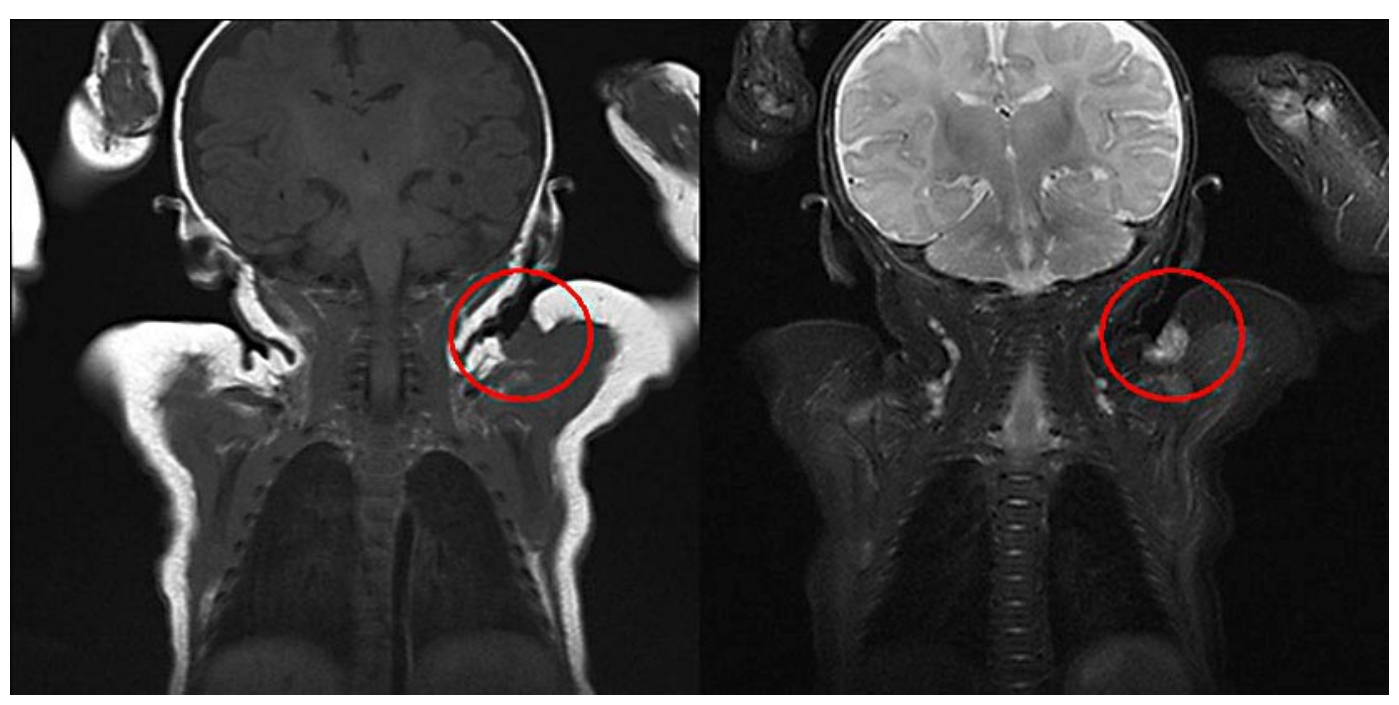

Fig. 2. MRI imaging showed the intensity of the nodule was similar to that of muscle. No additional lesions were found and infiltration of the tumor into the muscle was not observed.
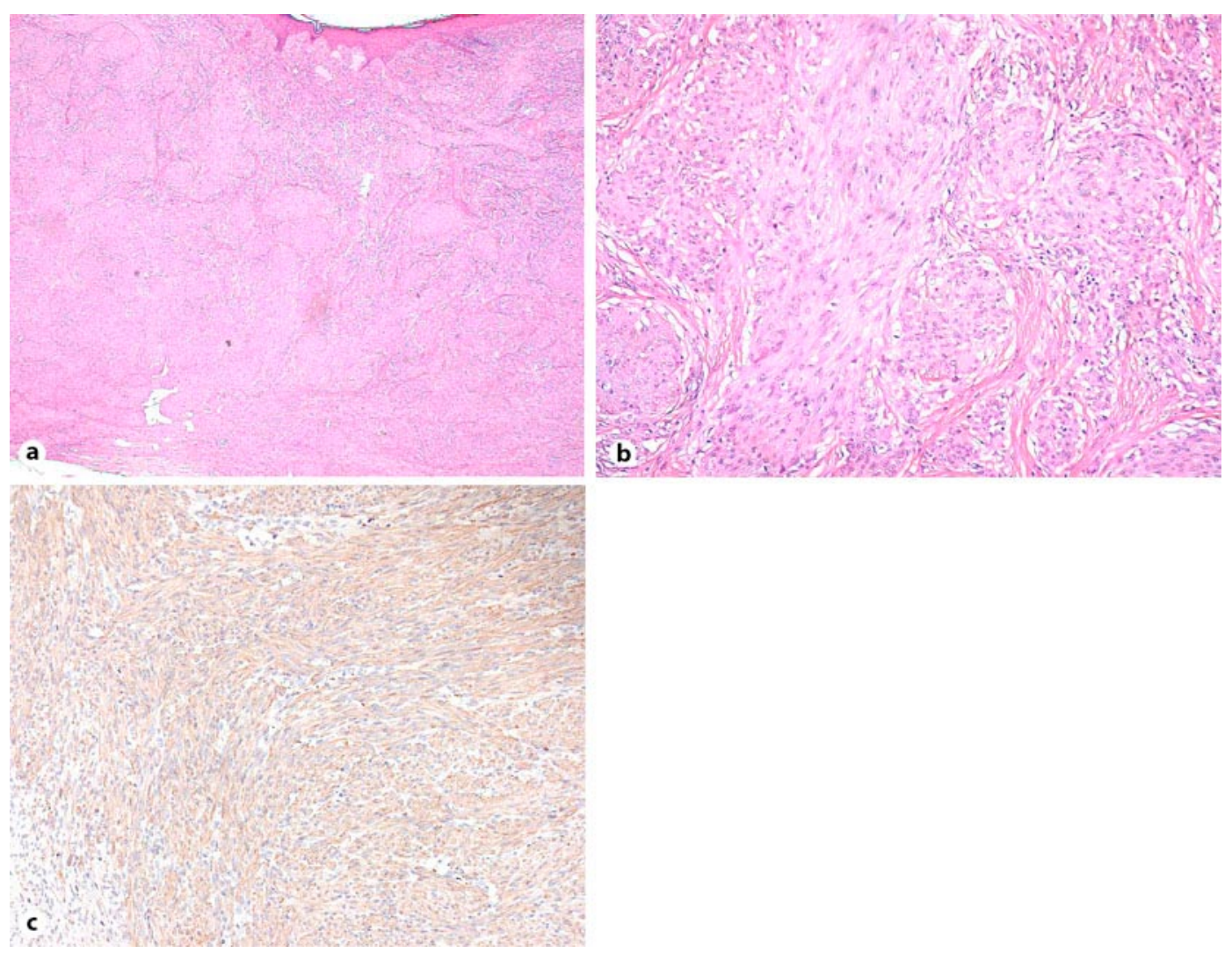

Fig. 3. Hematoxylin-eosin stain, original magnification $\times 20(\mathbf{a})$, and $\times 100(\mathbf{b})$. Specimen showed multifocal sclerotic dermal nodules composed of spindle cells and eosinophilic fibers. c Immunological staining of the tumor for $\alpha$-smooth muscle actin $(\times 100)$. Spindle cells express smooth muscle actin. 


\section{References}

1 Stout AP: Juvenile fibromatosis. Cancer 1954;7:953-978.

-2 Chung EB, Enzinger FM: Infantile myofibromatosis. Cancer 1981;48:1807-1818.

3 Larralde M, Hoffner MV, Boggio P, Abad ME, Luna PC, Correa N: Infantile myofibromatosis: report of nine patients. Pediatr Dermatol 2010;27:29-33.

4 Stanford D, Rogers M: Dermatological presentations of infantile myofibromatosis: a review of 27 cases. Australas J Dermatol 2000;41:156-161.

5 Goldberg NS, Bauer BS, Kraus H, Crussi FG, Esterly NB: Infantile myofibromatosis: a review of clinicopathology with perspectives on new treatment choices. Pediatr Dermatol 1988;5:37-46.

6 Hogan SF, Salassa JR: Recurrent adult myofibromatosis: a case report. Am J Clin Pathol 1992;97:810-814.

7 Wiswell TE, Sakas EL, Stephenson SR, Lesica JJ, Reddoch SR: Infantile myofibromatosis. Pediatrics 1985;76:981-984.

$\checkmark 8$ Ang P, Tay Y, Walford Y: Infantile myofibromatosis: a case report and review of the literature. Cutis 2004;73:229-231.

-9 Dictor M, Elner A, Andersson T, Fernö M: Myofibromatosis-like hemangiopericytoma metastasizing as differentiated vascular smooth-muscle and myosarcoma. Am J Surg Pathol 1992;16:1239-1247.

10 Granter SR, Badizadegan K, Fletcher CD: Myofibromatosis in adults, glomangiopericytoma, and myopericytoma: a spectrum of tumors showing perivascular myoid differentiation. Am J Surg Pathol 1998;22:513-525.

11 Weedon D: Tumors and tumor-like proliferations; in Weedon D (ed): Skin Pathology, ed 2. Philadelphia, Churchill Livingstone, 2002, pp 767-768.

12 Variend S, Bax NMA, van Gorp J: Are infantile myofibromatosis, congenital fibrosarcoma and congenital hemangiopericytoma histogenetically related? Histopathology 1995;26:57-62.

13 Fields JP, Helwig EB: Leiomyosarcoma of the skin and subcutaneous tissue. Cancer 1981;47:156-169.

14 Staut AP, Hill WT: Leiomyosarcoma of the superficial soft tissues. Cancer 1958;11:844-854.

15 Toren A, Perlman M, Polak-Charcon S, Avigad I, Katz M, Kuint Y, Rechavi G: Congenital hemangiopericytoma/infantile myofibromatosis: radical surgery versus a conservative 'wait and see' approach. Pediatr Hematol Oncol 1997;14:387-393. 